Received: April 01, 2017

Accepted: April 03, 2017

Published: April 13, 2017

\section{Fatal Hypocalcemia Due to Hungry Bone Syndrome af- ter Total Parathyroidectomy}

\author{
AmeyaHodarkar ${ }^{1}$,Nicole Yang ${ }^{1}$,Mervat Saleh ${ }^{1}$, Ahmad Abdin $^{1}$, Rachel Trippett ${ }^{2}$,Mazen O. \\ Al-Qadi ${ }^{3, *}$ \\ ${ }^{1}$ Department of Medicine, Memorial Hospital of Rhode Island, Alpert Medical School of \\ Brown University \\ ${ }^{2}$ Department of Family Medicine, Memorial Hospital of Rhode Island, Alpert Medical \\ School of Brown University \\ ${ }^{3}$ Section of Pulmonary and Critical Care Medicine, Yale-New Haven Hospital
}

*Corresponding author:Dr. Mazen O. Al-Qadi, MD, Section of Pulmonary and Critical Care Medicine, Yale-New Haven Hospital; E-mail: Mazen.al-qadi@ynhh.org

\section{Abstract}

\subsection{Introduction}

Mild and transient post-parathyroidectomy hypocalcemia is common and usually resolves within a week in most patients. In some cases, however, postoperative hypocalcemia is more severe and prolonged, despite normal or even elevated levels of parathyroid hormone (PTH). This phenomenon, called hungry bone syndrome, occurs in patients who have developed bone disease preoperatively due to a chronic increase in bone resorption induced by high levels of PTH. The underlying hyperparathyroidism can either be primary or secondary due to end-stage renal disease. Hypocalcemia may cause myocardial dysfunction with or without hypotension. Herein, we present a woman with hungry bone syndrome and hypocalcemia who progressed to cardiopulmonary arrest.

\subsection{Case Report}

A 52-year-old woman with a past medical history of end stage renal disease on hemodialysis, calciphylaxis, total parathyroidectomy with auto transplantation 4 years ago and parathyroidectomy (of remnant tissue) 2 weeks prior to presentation, presented with generalized weakness for 4 days and emesis for one day, associated with constipation. She had been receiving oral calcium 1000 mg four times a day along with 800 units of 1,25dihydroxyvitamin $\mathrm{D}$ at home after completing her postoperative recovery at the hospital. On admission, she was hypotensive with aortic systolic murmur and sluggish bowel sounds. Chvostek sign was negative.
EKG revealed sinus tachycardia and QTc prolongation. Initial lab work was significant for elevated creatinine, elevated serum phosphorus and severe hypocalcemia (total calcium $<4.0 \mathrm{mg} / \mathrm{dL}$ and an ionized $2.1 \mathrm{mg} / \mathrm{dL}$ ). The patient was dialysed with a high calcium bath. We refrained from giving IV calcium initially due to the risk of precipitating calciphylaxis. Post dialysis the calcium was $5.0 \mathrm{mg} / \mathrm{dL}$. The patient developed supraventricular tachycardia that was not responsive to adenosine and became hypotensive. She received norepinephrine for hemodynamic support and IV calcium was started. Four hours later, she developed asystole. CPR was performed for 8 minutes with return of spontaneous circulation. However, she had three more cardiac arrests in the next 24 hours. Due to the grim prognosis, code status was changed to "Do Not Resuscitate". Approximately 18 hours after the first code the patient had another asystole and passed away.

\subsection{Discussion}

Postparathyroidectomy hypocalcemia results from acute reversal of the effect of PTH on the bone. Sudden withdrawal of PTH in such patients causes an imbalance between bone formation and resumption affecting calcium fluxes, causing a marked increase in bone uptake of calcium, phosphate, and magnesium. Consequently the clinical features of hungry bone syndrome are due to hypocalcemia, hypophosphatemia, hypomagnesemia, and hyperkalemia. The serum calcium typically reaches a nadir two to four days postoperatively. Overt heart failure has been described in patients with myocardial dysfunction and typically resolves after normalization of the serum calcium. 
Careful monitoring of serum calcium is required to prevent and treat symptomatic hypocalcemia, which can be potentially catastrophic.

In our case, she presented out of the critical symptomatic window with vague symptoms suggestive of hypocalcemia.

Genetic analysis for the second sibling confirmed a novel mutation in the (SCNN1A) subunit gene of the (enac),

\subsection{Conclusion}

Hungry bone syndrome is a serious complication that can develop after parathyroidectomy. These patients should be closely monitored for serum ionized or total calcium levels with frequent outpatient labs. Hungry bone syndrome can present few weeks after the operation.

\section{Introduction}

Transient mild hypocalcemia following Parathyroidectomy is not uncommon and is reported in $13 \%$ of patients. This phenomenon, called the hungry bone syndrome (HBS), is primarily due to functional or relative hypoparathyroidism from reductions in bone re absorption and increase in bone formation, leading to an increased influx of calcium into bone. HBS usually resolves within a week .In some cases; however, the postoperative hypocalcemia is severe and prolonged, despite normal or even elevated levels of parathyroid hormone (PTH). Patients who have developed bone disease preoperatively (due to a chronic increase in bone resumption induced by high levels of PTH) are at higher risk of developing HBS [1,2]. The underlying hyperparathyroidism can either be primary or secondary due to endstage renal disease. Acute hypocalcemia has been known to cause myocardial dysfunction, poor myocardial performance and even congestive heart failure either with or without hypotension [3-8]. We herein present a woman with profound hypocalcemia due to hbswho progressed to cardiopulmonary arrest.

\section{Case Report}

A 52-year-old woman presented with generalized weakness for 4 days and emesis for one day. She also endorsed constipation and her last bowel movement was 5 days prior to presentation. The past medical history was significant for end stage renal disease (secondary to adult polycystic kidney disease, on thrice weekly hemodialysis for 6 years), hypertension, calciphylaxis, severe aortic stenosis, total parathyroidectomy with autotransplantation 4 years ago and parathyroidectomy (of remnant tissue) 2 weeks prior to presentation for severe symptomatic secondary hyperparathyroidism. She had been receiving oral calcium $1000 \mathrm{mg}$ four times a day along with 800 units of 1, 25dihydroxyvitamin $\mathrm{D}$ at home after completing her postoperative recovery at the hospital. Pre-operative PTH was $>1000 \mathrm{pg} / \mathrm{ml}$ (normal 10-65 pg/ml) for more than 4 years.

On admission, she was hypotensive at $93 / 57 \mathrm{mmhg}$ and tachypneic at 26 breaths/minute, but she was a febrile and with a normal heart rate. In addition, physical exam was significant for aortic systolic murmur, large ventral abdominal hernia, sluggish bowel sounds, but soft protuberant non-tender abdomen. Chvostek sign was negative and peripheral pulses were well felt. EKG revealed sinus tachycardia and qtc prolongation.

Initial lab work was significant for elevated Creatinine of $17.32 \mathrm{mg} / \mathrm{dl}$, elevated serum phosphorus of $11.8 \mathrm{mg} / \mathrm{dl}$ and normal lactate at $1.7 \mathrm{mmol} / \mathrm{L}$. Serum calcium was immeasurably low at $<4.0 \mathrm{mg} / \mathrm{dl}$ and an ionized calcium measured one hour later was also low at $2.1 \mathrm{mg} / \mathrm{dl}$. Liver function panel, thyroid function test and blood counts were within normal range. A chest radiograph showed no cardiopulmonary abnormalities, blood cultures were negative. The patient was dialysed with a high calcium bath. We refrained from giving IV calcium initially due to the risk of precipitating calciphylaxis.

Post dialysis the calcium was $5.0 \mathrm{mg} / \mathrm{dl}$. The patient developed supraventricular tachycardia which was not responsive to adenosine. Two hours later, she developed mottling of the skin and blood pressure was no longer measurable. She received norepinephrine for hemodynamic support and IV calcium was started. Despite aggressive resuscitative efforts and vasopressors, she developed asystole approximately 4 hours later. Cardiopulmonary resuscitation was performed for 8 minutes with return of spontaneous circulation. The patient developed asystole again $2 \mathrm{~min}$ utes later and received another round of cardiopulmonary resuscitation, following which she developed ventricular tachycardia. She was treated with intravenous magnesium, calcium and amiodarone and was emergently intubated and placed on mechanical ventilation.

A post-code right sided electrocardiogram revealed ST elevation in inferior leads. Her troponins rose to $0.498 \mathrm{ng} / \mathrm{ml}$. Owing to her hemodynamic instability, a cardiac catheterization was deferred and she was started on aspirin and intravenous heparin. Overnight, the patient had two pulseless electrical activity arrests. Due to the grim prognosis, the family decided to change the code status to "Do Not Resuscitate". Approximately 18 hours after the first code,she had another asystole and passed away.In the course of the hospitalization, the patient received 21 grams of intravenous calcium. Before her death her ionized calcium was found to be normal at $5.8 \mathrm{mg} / \mathrm{dl}$.

\section{Discussion}

The postoperative hypocalcemia probably results from acute reversal of the effect of PTH on the bone. In a high turnover state like hyperparathyroidism, PTH increases both bone formation and resumption with a net efflux of calcium from bone. Sudden withdrawal of PTH in such patients causes an imbalance between bone formation and resumption affecting calcium fluxes, causing a marked increase in bone uptake of calcium, phosphate, and magnesium [2]. Consequently, the clinical features of hungry bone syndrome are due to hypocalcemia, hypophosphatemia, 
hypomagnesemia, and hyperkalemia.The serum calcium typically reaches a nadir two to four days postoperatively [2]. Hypocalcemia resulting in overt heart failure has been described in patients with latent myocardial dysfunction where cardiomegaly and pulmonary congestion resolved after normalization of the serum calcium $[9,10]$. Careful monitoring two to four times per day for the first few postoperative days is required to prevent and treat symptomatic hypocalcemia, which can be potentially catastrophic. The measurement of ionized calcium is preferred to total serum calcium since the total serum calcium concentration may not reliably predict the ionized calcium concentration, especially among patients with chronic kidney disease.

Oral calcium supplementation (2 to $4 \mathrm{~g}$ of elemental calcium per day) should be started as soon as the patient is able to swallow. Intravenous calcium is indicated if the patient develops symptoms related to hypocalcemia, tetany, seizures, cardiac arrhythmias, laryngeal spasm, or either an ionized calcium concentration below $4 \mathrm{mg} / \mathrm{dl}$ or total serum calcium below $7.5 \mathrm{mg} / \mathrm{dl}$. Initially, intravenous calcium 1 to 2 grams of calcium gluconate can be infused over 10 to 20 minutes. This dose of calcium gluconate will raise the serum calcium concentration for only two or three hours. It should be followed by a slow infusion of calcium in patients with persistent hypocalcemia. Vitamin D supplementation is also useful, particularly in patients on maintenance dialysis who are often vitamin D deficient.

In the case of our patient, she had a total parathyroidectomy with removal of the auto-transplant two weeks prior to presentation. She had seemingly been on adequate calcium regimen while in the hospital and was discharged home on appropriate calcium and 1,25-dihydroxyvitamin D doses. She presented out of the critical symptomatic window with vague symptoms suggestive of hypocalcemia. Due to her initial presentation and history of calciphylaxis a decision was made to replete her calcium with dialysis rather than with IV boluses. The calcium was not given more rapidly, due to the risk of serious cardiac dysfunction, including systolic arrest [11]. Despite that the patient did develop tachyarrhythmias and progressive worsening in her blood pressure which eventually culminated into cardiopulmonary arrest.

This case brings to light two important aspects. First, the patient presented with hypocalcemia symptoms outside of what is considered the most critical window to develop HBS. The reason for this is not entirely clear, and possibly related to not being prescribed activated vitamin D. Second, this case highlights the amount of calcium deficit that can develop with severe cases of HBS. During the course of this time and 2 weeks post operation she had developed hypocalcemia which required 21 grams of intravenous calcium gluconate given gradually before the first normal ionized calcium was obtained.

\section{Conclusion}

HBS is a serious complication that can develop after parathyroidectomy. After postoperative repletion of calcium in the hospital, patients are typically discharged home with oral calcium and 1,25-dihydroxyvitamin D. These patients should be closely monitored for serum ionized or total calcium levels with frequent outpatient labs. HBS can present as symptomatic hypocalcemia even a couple of weeks after the operation. Perhaps more research is needed as to how aggressively to replete calcium in patients who present with a delayed onset of HBS.

\section{References}

1. Albright F, Reifenstein EC Jr. The parathyroid glands and metabolic bone disease, Williams Wilkins, Baltimore 1948;19(1):130-131.

2.Brasier AR, Nussbaum SR. Hungry bone syndrome: clinical and biochemical predictors of its occurrence after parathyroid surgery. Am J Med. 1988; 84(4):654-660.

3. Shinoda T, Aizawa T, Shirota T, katakura M, Yamada T, Chang YT, et al. Exacerbation of latent heart failure by mild hypocalcemia after parathyroidectomy in a long-term hemodialysis patient. Nephron. 1992;60(4):482-486.

4. Kazmi AS, Wall BM. Reversible congestive heart failure related to profound hypocalcemia secondary to hypoparathyroidism. Am J Med Sci. 2007;333(4):226-229.

5. Levine SN, Rheams CN. Hypocalcemic heart failure. Am J Med. 1985;78(6 Pt 1):1033-1055.

6. Wong CK, Lau CP, Cheng CH, Leung WH, Freedman B. Hypocalcemic myocardial dysfunction: short- and longterm improvement with calcium replacement. Am Heart J. 1990;120(2):381-386.

7. Kudoh C, Tanaka S, Marusaki S, Hayasi M, limura O,Kikuchi K, et al. Hypocalcemic cardiomyopathy in a patient with idiopathic hypoparathyroidism. Intern Med. 1992;31(4):561-568.

8. Brunvand L, Hågå P, Tangsrud SE, Haug E. Congestive heart failure caused by vitamin D deficiency? Acta Paediatr. 1995;84(1):106-108.

9. Falko JM, Bush CA, Tzagournis M, Thomas FB. Case report. Congestive heart failure complicating the hungry bone syndrome. Am J Med Sci. 1976;271(1):85-89.

10. Shinoda T, Aizawa T, Shirota T, yamada T, Chang YT, Arakura $\mathrm{H}$,et al. Exacerbation of latent heart failure by mild hypocalcemia after parathyroidectomy in a long-term hemodialysis patient. Nephron 1992;60(4):482-486.

11. Tohme JF, Bilezikian JP. Diagnosis and treatment of hypocalcemic emergencies. The Endocrinologist 1996;6(1):10. 